\title{
Retos en las Publicaciones Científicas en Estudiantes de Psicología
}

\author{
Challenges in Scientific Publications in Psychology Students
}

\author{
Yazmín Álvarez-Alatorre ${ }^{1}$ \\ 1 Ponce Health Sciences University, San Juan, Puerto Rico. (D) https:/orcid.org/0000-0001-8989-1679 \\ * Correspondencia: yalvarez19@stu.psm.edu
}

WWW.REVISTACARIBENADEPSICOLOGIA.COM

\section{Citar como:}

Álvarez-Alatorre, Y. (2022). Retos en las publicaciones científicas en estudiantes de psicología. Revista Caribeña de Psicología, 6, e6193. https://doi.org/10.37226/rcp.v6i1.6193

\section{Editorial Volumen 6, 2022}

El proceso de formación y conversión de un estudiante a un profesional competente es un gran reto para las universidades. La investigación científica inicia con una idea nueva que se estructura en forma de hipótesis, la cual será aceptada o rechazada según los resultados obtenidos; muchas veces este proceso culmina con la publicación de los hallazgos descubiertos. La preparación académica de los estudiantes en materia de investigación debe constituir parte de la formación curricular y extracurricular, para que de este modo puedan adquirir hábitos, cualidades y habilidades investigativas que se ajusten a los exigentes modelos de enseñanza de la actualidad (Hernández, 2018). El estudiante es comúnmente visto como un aprendiz y esta percepción puede que le quede corta o lo limite ante lo que realmente puede llegar a lograr en el campo investigativo. Siendo el motor de cambio de la sociedad y la base de la continuidad de las instituciones académicas, se debe replantear su rol en la proliferación y crecimiento de cualquier ciencia. Se debe procurar reducir esa tendencia a identificarlo como un ente pasivo en la investigación (Arbaiza-Bayona, 2012). Una de las principales vías para hacer público el conocimiento son las revistas científicas, que hoy en día constituyen uno de los más importantes medios de difusión de la ciencia (Aguado-López et al., 2008; Miguel, 2011), así como un excelente indicador del desarrollo académico (Krauskopf \& Vera, 1995). Sin embargo, la falta de una cultura de publicación ha hecho que la producción científica, principalmente en Latinoamérica, tenga poca difusión, visibilidad e impacto, y el alcance de los artículos (calculado por el número de citaciones) no logra competir con el de países más desarrollados (Miguel, 2011; Vera-Villarroel et al., 2011). Es por esto que, surgieron nuevos proyectos y políticas de acceso abierto, para eliminar esta disparidad y fomentar la distribución inmediata de la información (Aguado-López et al., 2008).

El proceso de formación estudiantil a nivel de pregrado y posgrado casi nunca incluye aspectos relacionados con el proceso de publicación (Arbaiza-Bayona, 2012). Por lo tanto, es importante promover la participación de los estudiantes en los procesos de publicaciones formales involucrándolos en aspectos de edición, redacción científica, búsqueda bibliográfica y metodología de investigación, entre otros (Mayta \& Peña-Oscuvilca, 2009). Ante esta experiencia, los estudiantes aumentarán la valoración de sus trabajos y sus competencias, además, adquirirán un criterio más amplio de la información a la que están expuestos y se darán cuenta que publicar es posible ya sea que participen como autores o como miembros del equipo editorial (Gutiérrez \& Mayta, 2003). 
Cabe mencionar, que muchas veces las revistas rechazan artículos por errores de redacción, presentación de datos, malos planteamientos del problema a investigar, metodologías deficientes o conclusiones injustificadas, por lo que se debe exhortar al estudiante a familiarizarse con las normas que rigen las publicaciones científicas, que en el caso de la psicología deben ser en el formato de la Asociación Americana de Psicología (APA, por sus siglas en inglés). Además, se debe tener en cuenta que la era de la información trajo consigo grandes cambios para los formatos físicos de libros y revistas, entre otros, por lo que se debe prestar especial atención a la nueva cultura de publicación en formato electrónico y las nuevas reglas y exigencias que demandan (Aguado-López et al., 2008).

Arbaiza-Bayona (2012) propone algunas herramientas para la construcción de una cultura de publicación a nivel estudiantil que incluye: (1) la motivación (fomentando el interés en el tema científico de la mano de la participación del docente como principal influencia y fuente de enseñanza), (2) la capacitación (tanto para ser autores o miembros del equipo editorial, como en temas de redacción, e implicaciones éticas del contenido) y (3) visibilización e impacto (implementando herramientas para que las revistas y publicaciones puedan tener reconocimiento local e internacional). Es importante que las universidades incluyan en su misión y visión particular, la formación de estudiantes investigadores. Sin la publicación de resultados, hallazgos y evidencias que generan los estudios de investigación, la ciencia en general no podría progresar. Esta es una razón más para que los estudiantes de psicología se involucren en la investigación y publiquen sus resultados.

Según Hernández et al. (2019), algunas limitaciones para la publicación estudiantil, que han llevado a una baja producción científica en estudiantes de pregrado de psicología son: (1) falta de valoración del trabajo realizado, (2) ausencia de una cultura de divulgación, y (3) escasa participación en las publicaciones (que en su mayoría son de tipo instrumental y expuestas mayormente en revistas estudiantiles). Estos autores proponen que para el aumento de publicaciones científicas estudiantiles se deben incrementar las horas dedicadas a investigar y motivarlos, retribuyendo, de algún modo, a aquellos que logren difundir los resultados de sus investigaciones en forma de artículo científico. Las universidades están llenas de estudiantes creativos, entusiasmados y deseosos por aprender; estas cualidades deben ser aprovechadas por aquellos docentes que, a través de la mentoría, puedan impulsar y motivar al estudiante a investigar $\mathrm{y}$, de ese modo, fortalecer el conocimiento científico (Jiménez-Chavez, 2018).

Hay estudios que señalan que la buena percepción de los estudiantes hacia la investigación, no necesariamente se relaciona con su actitud hacia la misma. Un reciente estudio realizado por Ortega et al. (2018) mostró que, aunque más del $90 \%$ de 358 estudiantes considera que la formación en investigación contribuye a su vida profesional y al 80\% les gustaría involucrarse en proyectos de investigación, tienen una escasa participación en eventos de divulgación científica. La actitud del estudiante dependerá de la motivación, condiciones de la institución (materiales y organizacionales) y de los procesos de enseñanzaaprendizaje por los que atravesaron. Es por esto que se debe mejorar el desarrollo de las capacidades del estudiante para la presentación de proyectos, e impulsar su participación en eventos de difusión científica. Además, resulta pertinente que los docentes expongan sus propios trabajos de investigación en sus clases haciendo énfasis en la aplicación del método científico. Por último, los autores recomiendan exigir y reforzar las normas de estilo de escritura científica en los trabajos académicos. Del mismo modo, los facultativos de programas graduados en psicología, a pesar de la importancia que le asignan a la publicación científica, reconocen la existencia de múltiples barreras que limitan ese proceso. La falta de tiempo y disciplina para escribir, las exigencias de su vida profesional y personal, la dificultad para encontrar colegas con intereses similares para colaborar en las publicaciones, la falta de adiestramiento y problemas con el dominio del idioma inglés, son algunos de los retos a los que se enfrentan (Lugo \& Bernal, 2009).

Existen prácticas que no fomentan ni motivan la realización de estudios de investigación por parte de los estudiantes ya que algunas universidades tienen la tendencia de otorgar títulos por medios distintos a la elaboración de tesis (Uribe et al., 2011). En Puerto Rico, por ejemplo, los programas de maestría en psicología de algunas universidades proveen la opción al estudiante de obtener el grado a través de tesis o examen de grado. 
Uno de los factores limitantes en el desarrollo de la investigación psicológica es la distribución desigual de los recursos para la investigación, con la probable causa de que el gobierno considere más valioso el aporte de la investigación de las ciencias naturales y biomédicas que el de la psicología y otras ciencias sociales. Esta situación de inequidad nos podría llevar a suponer que la producción de artículos científicos en estas materias, son mayores que las producidas en psicología (Lugo \& Bernal, 2009). Sin embargo, los datos estadísticos del Scimago Journal \& Country Rank (2021) para publicaciones científicas, ubican a Puerto Rico en el lugar número 68 a nivel mundial y en el 7 a nivel de Latinoamérica en cuanto a producción de artículos de psicología, a diferencia de Ciencias Biológicas (mundial 83, Latinoamérica 13) y Bioquímica, Genética y Biología Molecular (mundial 81, Latinoamérica 10), lo que contradice ese supuesto, ubicando a Puerto Rico a la vanguardia en sus aportes de diseminación científica en psicología.

Aun así, existen instituciones académicas e investigadores, en su mayoría independientes, que, a pesar de no tener la infraestructura de un centro de investigación, hacen valiosos aportes científicos que se traducen en beneficios para la sociedad en general. En una encuesta realizada a 33 facultativos de las principales instituciones académicas de programas graduados en psicología en Puerto Rico, realizada por Lugo y Bernal (2009), la gran mayoría catalogó como muy importante el publicar en revistas locales e internacionales y en revistas indexadas, así como presentar sus trabajos en foros locales e internacionales. Una entrevista en ese mismo estudio recopiló las impresiones de 17 facultativos con respecto a lo que consideran barreras y elementos facilitadores en el proceso de publicación. Algunas de las barreras que destacaron fueron la alta carga académica, la falta de fondos para la investigación, falta de asistentes de investigación, falta de énfasis en las publicaciones generadas por la facultad, falta de énfasis institucional en la investigación, entre otros. Dentro de las recomendaciones para facilitar la publicación, mencionaron la creación de incentivos económicos para fomentar este quehacer, proveer adiestramiento a la facultad en estrategias efectivas de publicación y motivar al estudiantado a escribir sobre sus tesis o disertaciones en colaboración con el profesor que les asesora, entre otras.
Según Zou et al. (2017), en los Estados Unidos, el promedio de publicaciones de un estudiante de psicología a nivel graduado es de $2.89(\mathrm{SD}=3.53)$. Estos autores aseguran que el número de publicaciones científicas puede influir en la futura obtención de un puesto de trabajo como facultativo en una universidad. Además, la participación en investigaciones científicas añade un valor adicional al entrenamiento profesional. Por ejemplo, la encuesta realizada a solicitantes para plazas de internado de la APPIC (Association of Psychology Postdoctoral and Internship Centers) explora la productividad en las publicaciones de artículos científicos al momento de la solicitud de prácticas (solo la mitad de los solicitantes tienen por lo menos 1 artículo revisado por pares, el 10\% tiene 5 y menos del 1\% tienen 15 o más) y cuando estas tasas se comparan podrían poner en ventaja de conseguir la plaza a aquellos con mayores publicaciones (Lund et al., 2016).

Se debe tener cuidado de no caer en el dilema de la cultura universitaria "publicar o perecer" (Harzing, 2011) y evitar que la búsqueda de la publicación de artículos en revistas reconocidas por su factor de impacto se vuelva obsesivo al punto de ver a los artículos científicos como la principal mercancía de una red de tráfico de conocimiento. Incluso, hay autores que hacen una advertencia a investigadores que puedan llegar a convertirse en esclavos del índice de impacto y que eventualmente puedan padecer la "patología de la búsqueda de información" y generar ansiedad, agobio e incertidumbre en el proceso (Santos \& Fernández-Ríos, 2016).

Los artículos que se publican en revistas científicas especializadas en psicología son de gran valor para la difusión del conocimiento y desarrollo de la profesión. Existen distintos tipos de artículos según el área o tema de interés, estos pueden ser editoriales, de comunicación breve, reportes de caso, investigativos, artículos originales, cartas al editor y de revisión, entre otros. De estos, los artículos de revisión no son siempre considerados para publicación (a menos que provengan de autores reconocidos), a pesar de que generalmente son más citados que otros tipos de artículos. Esta renuencia por parte de algunos consejos editoriales se debe, mayormente, a que una revisión no es tan sencilla ya que implica la realización de una síntesis del estado de investigación de un tema que debe incluir resultados actualizados de los estudios 
que se hayan hecho hasta el momento. Para que un artículo de revisión en el campo de la psicología aporte más que una sumatoria de investigaciones y un resumen (abstract) se deben seguir ciertas guías para su correcta redacción y elaboración. Así pues, un artículo de revisión por sí solo no proveerá una solución a las múltiples controversias que existen en el quehacer psicológico (donde no existen las verdades absolutas). Por lo tanto, el construirlos bien (en cuanto a redacción, contenido, referencias, conclusiones éticas críticas y socialmente relevantes) puede ser pieza clave y de inicio hacia la comprensión de la incierta y compleja realidad de los procesos psicológicos (Fernández-Ríos \& Buela-Casal, 2009).

\section{Conclusión}

El conocimiento es la mejor arma contra la ignorancia, pero ante un enemigo tan peligroso y que muchas veces se mimetiza con verdades a medias o mentiras camuflajeadas, se necesita un arma poderosa, la investigación. Es deber de todo aquel que produzca conocimiento basado en la evidencia, compartirlo. En una sociedad virtual donde los memes y las publicaciones de las redes sociales son las que "educan" y se dan por sentadas, se hace vital la promoción de la búsqueda de información verídica a través de plataformas fiables que contribuyan a la adquisición de un conocimiento actualizado, que a su vez sea detonante de la curiosidad investigativa, principalmente en los estudiantes.

La psicología es una ciencia compleja que en su desarrollo ha tenido grandes luchas y debates dualistas por estudiar procesos que divergen y convergen, que son tangibles e intangibles, como la mente y el cerebro o la conducta y las emociones. Cada una de esas luchas se ha combatido del mismo modo que se hace en el presente y que se hará en el futuro, proveyendo evidencia y sustentando toda teoría con investigación y no con opinión. La publicación de artículos en revistas especializadas en psicología es un tema que está cargado de mitos y realidades. Para empezar, la publicación de artículos no es privilegio de un sector de profesionales de la psicología. No se limita a aquellos con un doctorado orientado a la investigación (PhD) ni es exclusivo de aquellos que estén licenciados. Tampoco es más fácil publicar artículos siendo profesional que siendo estudiante, ni hay garantías de que un artículo será publicado por cierta revista en particular solo por poseer una hoja de vida impresionante. Todas estas características pueden influir, hasta cierto punto, pero no son definitivas para el éxito en el proceso de la publicación de un artículo.

Se ha comprobado que, tanto estudiantes como facultativos (en su mayoría doctores) de programas graduados en psicología, atraviesan por las mismas inquietudes ante el proceso de publicación de un artículo científico. Ambos grupos consideran que la investigación es parte fundamental tanto para el desarrollo profesional como para el avance de la psicología como ciencia, sin embargo, reconocen que hay barreras que limitan sus genuinas intenciones de aportar sus hallazgos a la ciencia. Una de las principales razones en las que coinciden tanto estudiantes como profesionales es en la falta de tiempo para dedicarle a la investigación debido a las demandas de sus respectivas labores. Las estadísticas señalan que por lo menos la mitad de los estudiantes a nivel doctoral que solicitan plaza para hacer prácticas de internado en Estados Unidos han publicado un artículo revisado por pares o menos (Lund et al., 2016).

Las investigaciones de diversos autores con respecto al tema de la publicación científica en el área de la psicología coinciden y basan sus recomendaciones en los argumentos de la mayoría de los participantes en sus muestras de estudio. Tanto estudiantes como profesionales sugieren una mayor capacitación en materia del proceso de publicación (técnicas de redacción según las normas APA y proceso de edición), mayor promoción de los eventos de investigación, mayores alianzas colaborativas entre pares, entre docente-estudiante y procurar buenos niveles de motivación.

Hacer accesible y atractivo el quehacer investigativo en la psicología, no es asignación de un solo ente. El estudiante necesita motivación y mentoría, del mismo modo que el profesional/facultativo necesita la provisión de herramientas necesarias para facilitar esta labor por parte de la institución universitaria que lo emplea. Por su parte, la Revista Caribeña de Psicología (RCP) reconoce estas necesidades, y a través de su plataforma de publicación científica continua, procura apoyar tanto al psicólogo en formación como al profesional. Es nuestra meta visibilizar el talento caribeño; es por esto que, los profesionales que ejercen su labor como revisores, integran pasión y 
compromiso en el proceso, siguiendo una meta que une a todos los psicólogos y que es uno de los fundamentos de la RCP: la búsqueda del conocimiento para entregarlo al bienestar de la humanidad.

\section{Novedades de la Revista}

En el 2021, la RCP realizó cambios en la periodicidad y velocidad de publicación de manuscritos. Desde el año pasado, la revista se publica de forma continua y los manuscritos sometidos serán publicados inmediatamente sean aceptados. Por tal razón, se mantendrá una convocatoria permanente para la recepción de artículos. Los artículos aceptados serán publicados inmediatamente al volumen correspondiente. El tiempo promedio de publicación de un manuscrito es entre 60 y 180 días desde que es recibido. Recordamos que los artículos deben someterse en formato Word y en estilo APA 7ma edición. Todo artículo debe ser sometido a través de nuestra página web. Les invitamos a seguir patrocinando la revista, tanto a los lectores asiduos como a los autores.

\section{REFERENCIAS}

Aguado López, Eduardo, Rogel Salazar, Rosario, Garduño Oropeza, Gustavo, \& Zúñiga, María Fernanda. (2008). Redalyc: Una alternativa a las asimetrías en la distribución del conocimiento científico. Ciencia, Docencia y Tecnología, 19(37), 11-30.

Arbaiza-Bayona, A. L. (2012). Revistas científicas estudiantiles de psicología en latinoamérica. Revista Colombiana de Psicología, 21(1), 151-164.

Fernández-Ríos, L., \& Buela-Casal, G. (2009). Standards for the preparation and writing of psychology review articles. International Journal of Clinical and Health Psychology, 9(2), 329-344.

Gutiérrez, C. \& Mayta, P. (2003). Publicación desde el pregrado en Latinoamérica: Importancia, limitaciones y alternativas de solución. CIMEL, 8(1), 54-60.

Harzing, A. W. (2011). The publish or perish book: Citation analysis for academics and administrators. Tarma Software Research.

Hernández-Moreno, V.J. (2018). La participación de los estudiantes en las publicaciones científicas. Medicent Electrón, 22(2), 2.

Hernández, R. M., Carranza Esteban, R. F., Caycho-Rodríguez, T.,
Cabrera-Orosco, I., \& Arias Chávez, D. (2019). Publicaciones científicas en revistas peruanas de psicología: un análisis desde la participación estudiantil. Revista Digital de Investigación en Docencia Universitaria, 13(2), 19-28.

Jiménez-Chaves, V. E. (2018). La formación de investigadores en la Universidad. ACADEMO Revista de Investigación en Ciencias Sociales y Humanidades, 5(1), 2.

Krauskopf, M. \& Vera, M. I. (1995). Las revistas latinoamericanas de corriente principal: indicadores y estrategias para su consolidación. Interciencia, 20(3), 144-148.

Lugo, E. A. \& Bernal G. (2009). El impacto científico de los programas graduados de psicología en Puerto Rico. Consejo de Educación Superior de Puerto Rico.

Lund, E. M., Bouchard, L. M., Thomas, K. B. (2016). Publication productivity of professional psychology internship applicants: An in-depth analysis of APPIC survey data. Training and Education in Professional Psychology, 10(1), 54-60.

Mayta, P. \& Peña-Oscuvilca, A. (2009). Importancia de la publicación en las sociedades científicas de estudiantes de medicina del Perú: estudio preliminar. CIMEL, 14, 27-34.

Miguel, S. (2011). Revistas y producción científica en América Latina y el Caribe: Su visibilidad en Scielo, Redalyc y Scopus. Revista Interamericana de Bibliotecología, 34(2), 187-198.

Ortega Carrasco, R. J., Veloso Toledo, R. D., \& Hansen, O. S. (2018). Percepción y actitudes hacia la investigación científica. ACADEMO Revista de Investigación en Ciencias Sociales y Humanidades, 5(2), 101-109. http://dx.doi.org/10.30545/academo.2018.jul-dic.2

Santos-Rego M. A., \& Fernández-Ríos, L. (2016). El factor de impacto y el futuro de las revistas académicas: El riesgo de patologización. Innovación Educativa, 16(72), 35-51.

Scimago Journal \& Country Rank. (2021). https://www.scimagojr.com/journalrank.php

Uribe Alvarado J. (2011). Percepción de la Investigación científica e intención de elaborar tesis en estudiantes de psicología y enfermería. Enseñanza e Investigación en Psicología, 16(1), 15-26.

Vera-Villarroel, P., López-López, W., Lillo, S., \& Silva, L. M. (2011). La producción científica en psicología latinoamericana: Un análisis de la investigación por países. Revista Latinoamericana de Psicología, 43(1), 95-104.

Zou, C. (2017). The publication trajectory of graduate students, post-doctoral fellows, and new professors in psychology. Scientometrics, 117(2), 1289-1310. https://doi.org/10.1007/s11192-017-2540-6 